\title{
Liquid metal nanodroplet dynamics inside nanocontainers
}

SUBJECT AREAS:

NANOSCIENCE AND

TECHNOLOGY

STRUCTURE OF SOLIDS AND

LIQUIDS

PARTICLE PHYSICS

NANOSCALE MATERIALS

Received

29 May 2013

Accepted

14 August 2013

Published

5 September 2013

Correspondence and requests for materials should be addressed to P.M.A. (pma2@rice. edu) or Y.J.J. (jungy@ coe.neu.edu)

\author{
Hyun Young Jung', Hyunkyung Chun', Sora Park², Seoung-Hun Kang' ${ }^{2}$, Chi Won Ahn ${ }^{3}$, \\ Young-Kyun Kwon'2, Moneesh Upmanyu', Pulickel M. Ajayan' \& Yung Joon Jung',2
}

'Department of Mechanical and Industrial Engineering, Northeastern University, Boston, Massachusetts, 0211 5, USA, ${ }^{2}$ Department of Physics and Research Institute for Basic Sciences, Kyung Hee University, Seoul, 130-701, Korea, ${ }^{3}$ Nano-Materials Lab, National NanoFab Center, Korea Advanced Institute of Science and Technology, 53-3 Eoeun-dong, Yuseong-gu, Daejeon, 305-806, Republic of Korea, ${ }^{4}$ Department of Mechanical Engineering and Material Science, Rice University, Houston, Texas, 77005, USA

Here we report direct observations of spatial movements of nanodroplets of $\mathrm{Pb}$ metal trapped inside sealed carbon nanocontainers. We find drastic changes in the mobility of the liquid droplets as the particle size increases from a few to a few ten nanometers. In open containers the droplet becomes immobile and readily evaporates to the vacuum environment. The particle mobility strongly depends on confinement, particle size, and wetting on the enclosed surface. The collisions between droplets increase mobility but the tendency is reversed if collisions lead to droplet coalescence. The dynamics of confined nanodroplets could provide new insights into the activity of nanostructures in spatially constrained geometries.

$\mathrm{n}$ recent years, there have been significant efforts to visualize and understand physical behaviors of nanoparticles under various thermodynamic and interfacial conditions using in-situ transmission electron microscopy $(\mathrm{TEM})^{1-8}$. A nanometer sized particle has a large surface area to volume ratio, which leads to significantly different thermodynamic properties such as phase coexistence and melting below the bulk melting temperature ${ }^{9-15}$. Nanoparticles when constrained also show confinement effects due to the interaction between nanoparticles and the confining environment ${ }^{16-22}$. For instances, due to the strong C-C covalent bonds, closed-shell carbon nanostructures such as carbon onion and carbon nanotubes have been shown to act as self-contracting high-pressure $(40-100 \mathrm{GPa})$ cells under the electron irradiation resulting in drastically altered structures, shapes, and phases $^{23-25}$. It still remains as a challenge to directly visualize the behavior of nanoparticles in confinement under various thermodynamic conditions. Here we report the direct observation of dynamic motions of molten lead $(\mathrm{Pb})$ nanoparticles in confined space provided by carbon nanocontainers, using in-situ TEM, and show random collective motions of $\mathrm{Pb}$ nanodroplets driven by collisions and Oswald ripening.

\section{Results}

Formations of nanodroplets inside nanocontainer. Our approach to create $\mathrm{Pb}$ nanoparticles inserted nanocontainer system is shown schematically in Fig. 1a. The nanocontainer, in the form of a carbon nanocup engineered from graphitic carbon, was fabricated by using precisely controlled short nanopores inside anodic aluminum oxide templates and thermal chemical vapor deposition of acetylene $\left(\mathrm{C}_{2} \mathrm{H}_{2}\right)$ at $650^{\circ} \mathrm{C}^{26,27}$. As shown in scanning electron microscope (SEM) and transmission electron microscopy (TEM) images (Fig. 1b and 1e), the open nanoscale cup geometry as well as its graphitic nature make carbon nanocups a robust nanocontainer which prevent the change in volume and interfacial area during heating. Differently sized $\mathrm{Pb}$ nanoparticles were formed inside the nanocontainer by controlling $\mathrm{Pb}$ deposition. The free space inside the nanocontainer and the particle size determines the motion of the nanodroplets that form upon heating. For typical figures, as shown in Fig. 1c-1d and $1 \mathrm{f}-1 \mathrm{~g}$, the ratio of the particle volume to free volume ranges from $1: 4.8$ to $1: 310$ for the large $(60 \mathrm{~nm})$ to the small $(15 \mathrm{~nm})$ droplets we have observed. Finally, a carbon film $\left(7 \mathrm{~nm}\right.$ thickness) was deposited under $10^{-6}$ torr pressure to cap the nanocontainer making it a perfectly sealed, closed system.

Observation of nanodroplets by in-situ TEM. In-situ transmission electron microscopy (JEOL-3011 with an accelerating voltage of $300 \mathrm{kV}$ equipped with a heating specimen stage) studies were performed to observe the motion of $\mathrm{Pb}$ nanodroplets inside the sealed nanocontainers. The specimen temperature was increased to $\sim$ $630 \mathrm{~K}$ for a large $\mathrm{Pb}$ nanoparticle $(\sim 60 \mathrm{~nm}$ diameter $)$ and $600 \mathrm{~K}$ for small $\mathrm{Pb}$ nanoparticles $(\sim 15-20 \mathrm{~nm}$ diameters), in order to undergo complete phase transition from solid to liquid (confirmed by molecular 


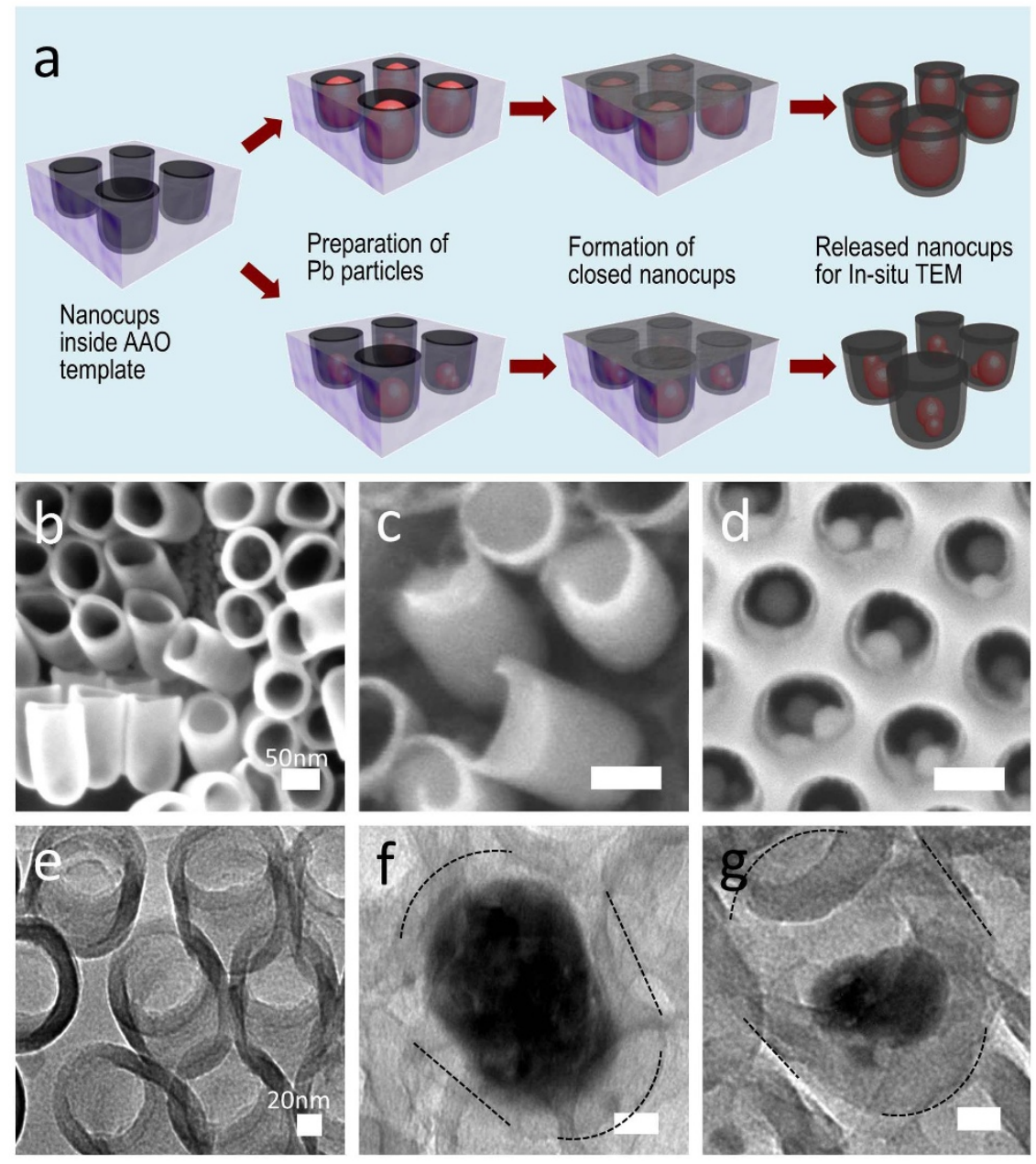

Figure 1 Schematic of sample preparation, and SEM and TEM images. (a) Schematic illustrating overall process to make closed nanocup container systems which are filled with different sizes of $\mathrm{Pb}$ nanoparticles. First nanocups were prepared inside AAO template by CVD and Ar ion milling processes. Next $\mathrm{Pb}$ nanoparticles were formed inside nanocontainers by thermal evaporation and annealing process. The size of $\mathrm{Pb}$ nanoparticles was controlled by adjusting evaporation time. Finally the nanocontainers with $\mathrm{Pb}$ nanoparticles were sealed with carbon layer on the open end, resulting closed nanocup system. (b-d) SEM images and (e-g) TEM images: (b) and (e) carbon nanocups fabricated after Ar ion milling on connected nanocups; (c) and (f) large size Pb nanoparticles formed inside nanocontainers; (d) and (g) small size Pb nanoparticles. TEM images were taken after tilting TEM holder by $30^{\circ}$ in $\mathrm{x}$-axis showing side view of the system structure. Dotted line indicates nanocontainer structure with sealing cap. Scale bars are $50 \mathrm{~nm}$ for SEM images and $20 \mathrm{~nm}$ for TEM images, respectively.

dynamic simulations, supplemental text and Fig. S1). Fig. 2 is the sequential TEM images of $\mathrm{Pb}$ nanodroplets captured over a period of $1-5$ seconds at the elevated temperature. At the early stage of heating, a melted larger $\mathrm{Pb}$ nanoparticle starts to fluctuate in both axial and radial directions inside the cylindrical nanocup (Fig. 2a and supplemental movie S1). This fluctuation occurred in random directions due to the limited free space as well as the interfacial forces between the nanocontainer surface and the molten $\mathrm{Pb}$ nanodroplet. With time, this motion becomes more along the axial direction of the nanocontainer following the cylindrical empty space that exists in the nanocup container (supplemental Fig. S2 and movie S1). During heating and melting of particles, we also observed that irregularly shaped $\mathrm{Pb}$ nanoparticles changed the shape to near spherical without the movement reflecting the lowest surface energy/volume ratio at thermodynamic equilibrium for the isotropic molten particle.

Unlike larger confined $\mathrm{Pb}$ nanodroplets, smaller $\mathrm{Pb}$ nanodroplets showed significantly different dynamic behavior inside the nanocontainer (Fig. 2b-c). Smaller droplets ceaselessly moved inside the nanocontainer as shown in Fig. 2b-c and supplemental movie S2. Individual movement of each nanodroplet appeared random and at a much faster rate than the motion of the larger droplets seen in Fig. 2a.
In addition to the brisk movements of the small nanodroplets we also observed spontaneous evaporation of 1-3 nm sized secondary droplets from the volume of the $\mathrm{Pb} 15-20 \mathrm{~nm}$ droplets, and the coalescence between them; despite the fact that smaller nanodroplets had active movement inside the nanocontainer, collisions and coalescence between two such droplets was only observed rarely.

\section{Discussion}

To understand the movement of smaller $\mathrm{Pb}$ nanodroplets inside nanocontainer, we monitored their position in 2-dimensional space and mean square displacement (MSD) over time as shown in Fig. 3 and supplemental movie S2. Two dimensional projected position changes of the two nanodroplets (Fig. 2b) and the merged droplet (Fig. 2c) are shown in Fig. 3a and b. The positions were marked every 0.1 seconds. The two nanodroplets moved irregularly in the nanospace, frequently changing their direction without overlapping displacements before being merged. This nonoverlapping might be due to the electrostatic charging effect by primary electron absorption from TEM into nanodroplets. Since each of two nanodroplets occupies smaller space than merged, the scaling with particle size is counter-intuitive as one would expect the smaller droplet reaching 

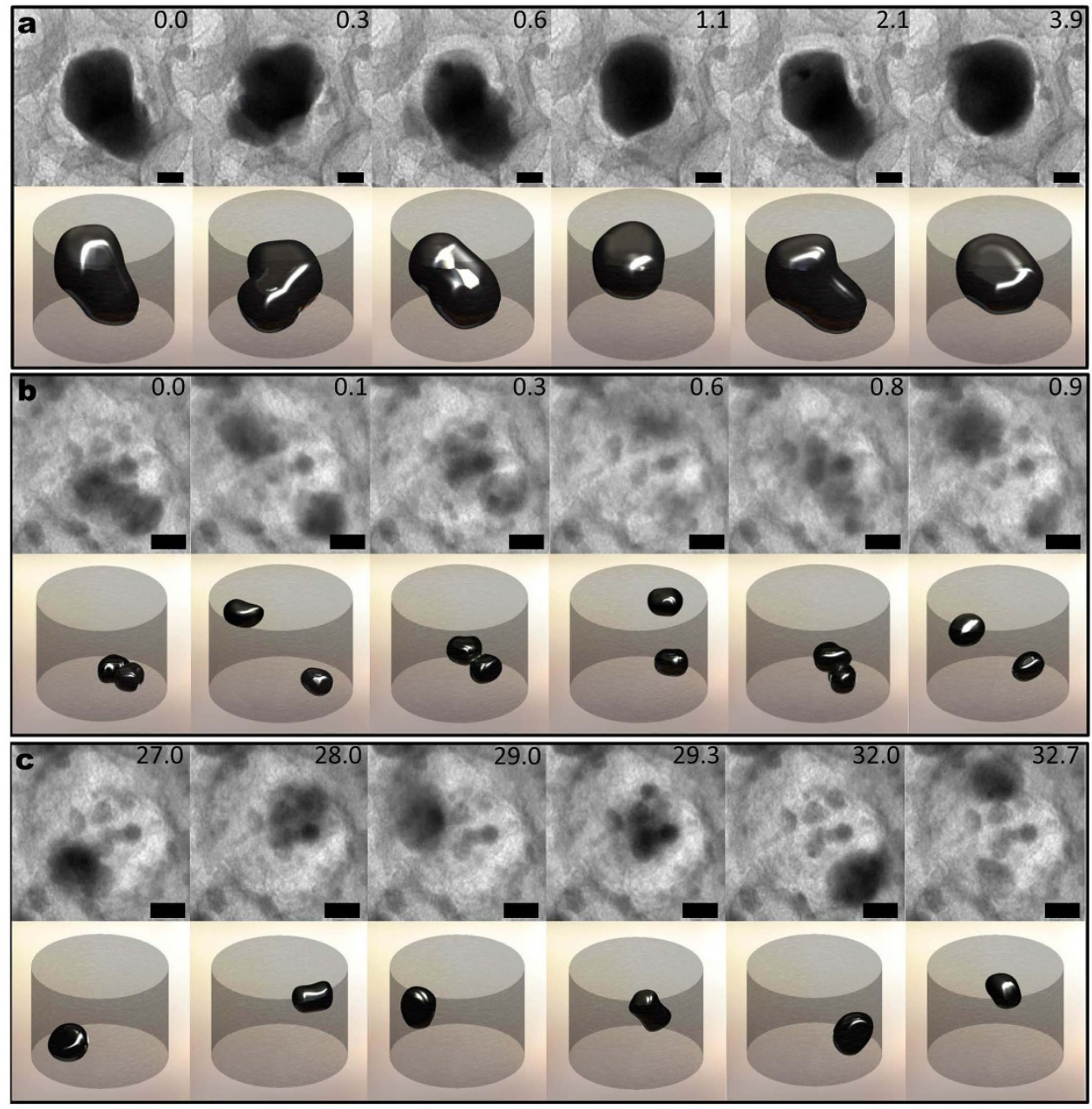

Figure $2 \mid$ The dynamic motions and schematics of a large $\mathrm{Pb}$ nanodroplet, two small $\mathrm{Pb}$ nanodroplets, and a merged droplet. TEM images were taken after tilting TEM holder by $30^{\circ}$ in $\mathrm{x}$-axis. The schematics indicate the positions of each particle over time in three-dimensional space. Dynamic motion of (a) large size $\mathrm{Pb}$ nanodroplet at $630 \mathrm{~K}$ inside the nanocontainer, (b) Two Pb nanodroplets inside nanocontainers at $600 \mathrm{~K}$, and (c) the merged droplet. The small droplets move randomly but are finally merged to one droplet due to collision. All scale bars are $20 \mathrm{~nm}$.

to a much larger asymptotic values. For the smaller droplets (Fig. 2b) and the merged slightly larger one (Fig. 2c), the second temporal evolution is seen as diffusive (Fig. 3c). A linear increase in the MSD is indicative of Brownian dynamics of the droplet. We speculate that upon heating under low pressure, surface atoms in nanodroplets are energetically excited and evaporated from the nanodroplets. Simultaneously these evaporated clusters can be condensed on the wall of nanocontainer. When condensed droplets become bigger than evaporated clusters, the vapor pressure become smaller again but the confined system will be placed in a nonequilibrium state and droplets will evaporate and condense repeatedly. In this case, the nanodroplet motion is influenced by condensation and evaporation of clusters, van der Waals attractive forces with the container, and unlike most systems, is not mediated by a solvent or by gas molecules. Thus we conjecture that the observed irregular motions of nanodroplets are due to the transfer of energy and momentum from the clusters to the nanodroplets through amplified molecular collisions inside of nanoscale confined space. Future study will be necessary to quantitatively understand the dynamics of nanoparticles/droplets in confined systems, which will provide important insight nanoscale influences on melting point, vapor pressure, and heat transfer.

As further validation of the origin of the collective diffusive behavior of the confined nanodroplet, we have characterized the nanodroplet behavior after the capping layer is punctured. Carbon nanocontainers have strong $\mathrm{C}-\mathrm{C}$ bonds and multi-layered graphitic structure, while the cap of nanocups was created by depositing amorphous carbon, which can be cracked by an external force. Fig. 4 and supplemental movie 33 shows the shape changes of a liquid $\mathrm{Pb}$ nanodroplet after the cap of the nanocontainer is punctured. The stationary liquid $\mathrm{Pb}$ droplet at thermodynamic equilibrium abruptly moved toward the end (sealing) of the nanocontainer after 0.03 seconds (Fig. 4a and b and supplement movie S4). This phenomenon is due to the pressure difference of inside and outside (under $10^{-7}$ torr in TEM chamber) of the nanocup container. The thermal energy is used to transform a given quantity of a substance from a liquid into a gas at a given pressure. The pressure and temperature within the nanocontainer rapidly equilibrates to the ambient conditions inside the vacuum column of the TEM. Under these conditions the vapor pressure of the metal changes abruptly. $2 \mathrm{D}$ characterization of the nanodroplet at several temperatures above the melting point shows that static nanodroplets wet the nanocontainer sidewalls with well-defined contact angles. Increasing temperature results in enhanced evaporation and the droplet reduces in size. The evaporation kinetics is discontinuous due to the heterogeneous nature of the defective sidewalls of the container. At larger volumes, the nanodroplet develops a meniscus as it wets multiple sidewalls. Below a critical droplet size, the volume becomes low enough such that the droplet is forced to wet a single sidewall and as a result, the droplet surface curvature changes sign. Supplemental movie S3 of the evaporation kinetics indicates a transition between pinned and moving contact 


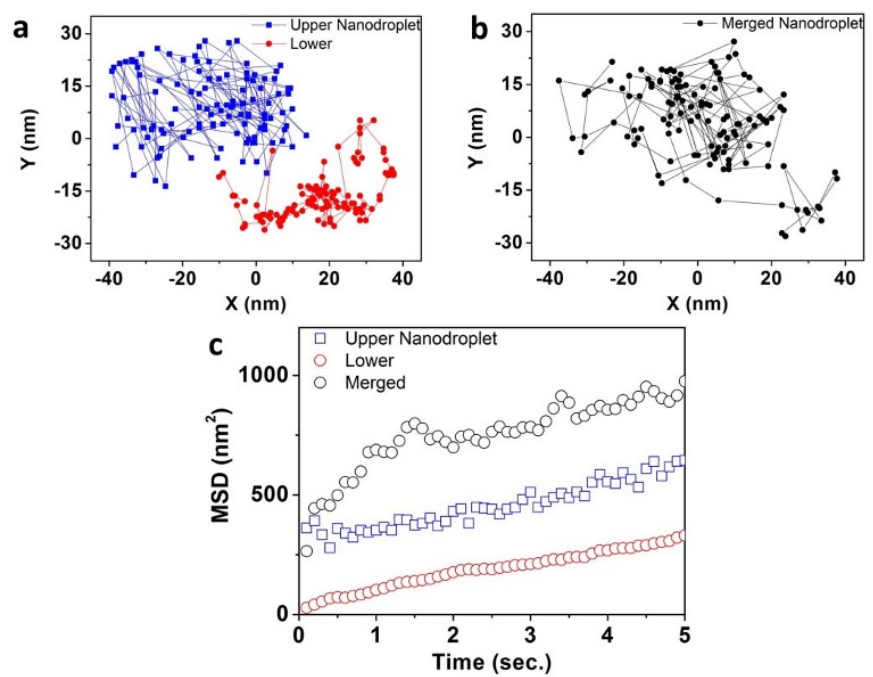

Figure $3 \mid$ Position trace and the mean square displacement of the centerof-mass of the lead nanodroplet. Two dimensional projected position changes of the two nanodroplets (a) and the merged droplet (b). The positions were marked every 0.1 seconds. The two nanodroplets move irregularly without overlapping displacements before being merged.

(c) Temporal evolution of the mean square displacement of the center-ofmass of the two lead nanodroplets (upper and lower one in Fig. 2(b)) and of the eventual merged droplet (Fig. 2c) over second time resolution. The linear behaviors indicate Brownian dynamics of the particle.

line regimes. This is also evident in Figure 4j, which shows the evaporation tendency of the instantaneous area of cross-section of the droplet at spontaneously increased temperatures. The area for the nanodroplet decreases with increasing temperature. The rate increases rapidly between 770 and $820 \mathrm{~K}$ where its shape is changed concave to convex.

In conclusion, we have observed the differences in the mobility of nanodroplets of molten metal trapped inside closed nanospaces and found dramatic differences between the mobility and diffusive nature of these particles as a function of particle size, wetting and confinement. The particles remain active only within closed environments and once open to the environment remains vulnerable to quick evaporation. Although transferable energy of incident electrons in TEM can contribute to droplet motion (shown in the supplemental text, Fig. S4 and movie S5), the dominant factor contributing to the motion is thermal activation that is causative of continuous collisions of the clusters onto the nanodroplets. The results show that under confinement, nanodroplets remain extremely mobile and undergo Brownian movements in the confined space. This could be potentially important in the understanding of the effect of confinement in the activity of small liquid droplets in environments such as porous media.

\section{Methods}

Formations of nanodroplet inside nanocontainer. Nanoporous alumina template was prepared using a standard electrochemical anodization process to fabricate highly organized short nanochannels of $120 \mathrm{~nm}$ in length. The connected arrays of carbon nanocup film structure were synthesized by using a chemical vapor deposition (CVD) process. Ar ion-milling process was then used in order to fabricate length controlled and separated nanocontainer structures. The size of $\mathrm{Pb}$ nanoparticles inside nanocontainers was controlled by adjusting the thickness of a $\mathrm{Pb}$ film deposited by thermal evaporation and by being annealed at $500^{\circ} \mathrm{C}$ for $5 \mathrm{hrs}$ in $\mathrm{Ar}$ atmospheric environment (780-850 Torr). For sealing of $\mathrm{Pb}$-inserted nanocup container system, $7 \mathrm{~nm}$ thick amorphous carbon film was deposited on the open area of nanocup by Precision Etching and Coating System (PECS, Gatan Inc.) under $10^{-4}$ torr pressure.

In-situ TEM. In-situ TEM studies of the $\mathrm{Pb}$ nanoparticles behavior inside the carbon nanocontainers were carried out in a JEOL-3011 with an accelerating voltage of $300 \mathrm{kV}$ equipped with a heating specimen stage. Double tilt heating holder was used for placing sample and it was tilted by $30^{\circ}$ in $\mathrm{x}$-axis during imaging for all
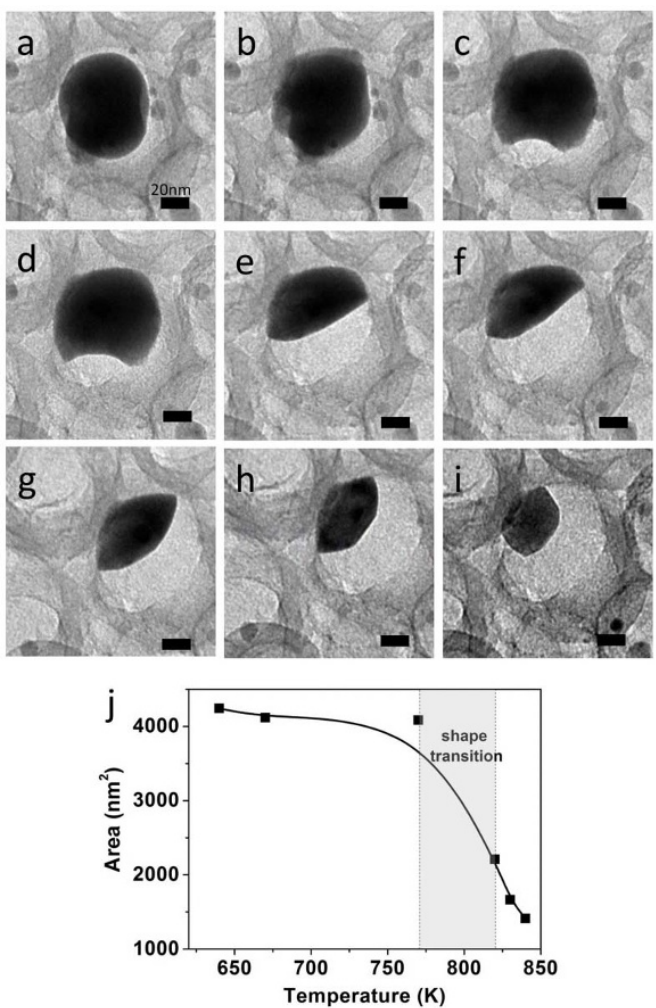

Figure 4 The behavior of $\mathrm{Pb}$ nanodroplet in the open nanocontainer. The size of $\mathrm{Pb}$ nanodroplet gradually decreases due to evaporation through the open space. (a) Images at $640 \mathrm{~K}$. The motion of liquid $\mathrm{Pb}$ nanodroplet stopped and shape of liquid droplet became spherical. (b) After 0.03 seconds, this stationary liquid $\mathrm{Pb}$ droplet moved toward the end (sealing) of the nanocontainer (temperature is $640 \mathrm{~K}$ ). (c) and (d) Image at $670 \mathrm{~K}$ and $770 \mathrm{~K}$, respectively. The shape of liquid $\mathrm{Pb}$ changed to a hollow, concave shape. (e)-(i) Images at $820 \mathrm{~K}, 825 \mathrm{~K}, 830 \mathrm{~K}, 835 \mathrm{~K}$ and $840 \mathrm{~K}$, respectively. ( $\mathrm{j}$ ) The evaporation tendency of the instantaneous area of cross-section of the droplet at spontaneously increased temperatures. The size of liquid $\mathrm{Pb}$ nanodroplet slowly decreased due to evaporation with increasing temperature through the open space. The rate increased rapidly between 770 and $820 \mathrm{~K}$ and its shape eventually changed concave to spherical. Scale bars are $20 \mathrm{~nm}$.

experiments. All specimens were placed on holey carbon coated molybdenum grid contacted to the heating stage having the electric heater and heated to experimental temperature.

Computational method. To describe the thermodynamic properties of $\mathrm{Pb}$ nanoclusters as well as the bulk $\mathrm{Pb}$ structure, we performed molecular dynamics (MD) simulations within canonical ensemble using the first-principles DFT. We solved the classical equation of motion with the force acting on each atom, which was calculated using the Hellmann-Feynman force theorem. To prepare the canonical ensemble, we controlled the equilibrium temperature.

We used the Perdew-Zunger form ${ }^{28}$ of the Ceperley-Alder exchange-correlation functional ${ }^{29}$ in the local-density approximation to DFT, as implemented in the SIESTA $\operatorname{code}^{30}$. The behavior of valence electrons was described by norm-conserving Troullier-Martins pseudopotentials ${ }^{31}$ in the Kleinman-Bylander factorized form ${ }^{32}$ including the scalar-relativistic effect. An atomic orbital basis with a double- $\zeta$ polarization was used to expand the electronic wave functions. The charge density and potentials were determined on a real-space grid with a mesh cutoff energy of 200 Ry. We used a confinement energy shift of $0.01 \mathrm{eV}$, which defines the cutoff radii of the atomic orbitals. For the bulk Pb structure, the cubic supercell of 32 atoms was constructed in face-centered cubic (fcc) structure with a periodic boundary condition. We constructed $\mathrm{Pb}$ nanoparticles with 55 and 13 lead atoms.

For the investigation of the phase transition, we calculated the response of a canonical ensemble of $\mathrm{Pb}$ nanoparticles and fcc bulk structure to gradually increasing heat bath temperatures using Nosé thermostat ${ }^{33-35}$ and these simulations were carried out for 10 ps with a chosen time step of $1 \mathrm{fs}$.

1. Scholl, J. A., Koh, A. L. \& Dionne, J. A. Quantum plasmon resonances of individual metallic nanoparticles. Nature 483, 421-427 (2012). 
2. Rodney, S. R., Donald, C. L., Bryan, C., Ripudaman, M. \& Shekhar, S. Single crystal metals encapsulated in carbon nanoparticles. Science 259, 346-348 (1993).

3. Xia1, Y. et al. Self-assembly of self-limiting monodisperse supraparticles from polydisperse nanoparticles. Nat. Nanotech. 6, 580-587 (2011).

4. Kim, B. J. et al. Kinetics of individual nucleation events observed in nanoscale vapor-liquid-solid growth. Science 322, 1070-1073 (2008).

5. Yuk, J. M. et al. High-resolution EM of colloidal nanocrystal growth using graphene liquid cells. Science 336, 61-64 (2012).

6. Christian, C. Watching solution growth of nanoparticles in graphene cells. Science 336, 44-45 (2012).

7. Holmberg, V. C., Panthani, M. G. \& Korgel, B. A. Phase transitions, melting dynamics, and solid-state diffusion in a nano test tube. Science 326, 405-407 (2009).

8. Ajayan, P. M. \& Marks, L. D. Evidence for sinking of small particles into substrates and implications for heterogeneous catalysis. Nature 338, 139-141 (1989).

9. Schmidt, M., Kusche, R., Issendorff, B. \& Haberland, H. Irregular variations in the melting point of size-selected atomic clusters. Nature 393, 238-240 (1998).

10. Ajayan, P. M. \& Marks, L. D. Quasimelting and phases of small particles. Phys Rev. Lett. 60, 585-587 (1988).

11. Landman, U. E., Montroll, W. \& Shlesinger, M. F. Motion of clusters on surfaces. Phys. Rev. Lett. 38, 285-289 (1976).

12. Alloyeau, D. et al. Size and shape effects on the order-disorder phase transition in CoPt nanoparticles. Nat. Mater. 8, 940-946 (2009).

13. Navrotsky, A., Ma, C., Lilova, K. \& Birkner, N. Nanophase transition metal oxides show large thermodynamically driven shifts in oxidation-reduction equilibria. Science 330, 199-201 (2010).

14. Hosokawa, M., Nogi, K., Naito, M. \& Yokoyama, T. Nanoparticle technology handbook. Amsterdam: Elsevier (2007).

15. Kang, J. \& Kim, Y. H. Half-solidity of tetrahedral-like $\mathrm{Al}_{55}$ clusters. ACS Nano 4, 1092-1098 (2010).

16. Heuberger, M., Zäch, M. \& Spencer, N. D. Density fluctuations under confinement. Science 292, 905-908 (2001).

17. Cleveland, C. L. \& Landman, U. Dynamics of cluster-surface collisions. Science 257, 355-361 (1992).

18. Taverna, D. et al. Probing physical properties of confined fluids within individual nanobubbles. Phys. Rev. Lett. 100, 035301 (2008).

19. Han, Y. et al. Brownian motion of an ellipsoid. Science 314, 626-630 (2006).

20. Yokota, T., Howe, J. M., Jesser, W. A. \& Murayama, M. Fractional brownian motion of an $\mathrm{Al}$ nanosphere in liquid Al-Si alloy under electron-beam irradiation. J. Appl. Phys. 95, 5756-5761 (2004).

21. Johnson, E. et al. Brownian motion of liquid lead inclusions along dislocations in aluminum. J. Mater. Sci. 40, 3115-3119 (2005).

22. Burada, P. S. et al. Diffusion in confined geometries. ChemPhysChem 10, 45-54 (2009).

23. Sun, L. et al. Carbon nanotubes as high-pressure cylinders and nanoextruders. Science 312, 1199-1202 (2006).

24. Banhart, F., Hernández, E. \& Terrones, M. Extreme superheating and supercooling of encapsulated metals in fullerenelike shells. Phys. Rev. Lett. 90 , 185502 (2003)

25. Banhart, F. \& Ajayan, P. M. Carbon onions as nanoscopic pressure cells for diamond formation. Nature 382, 433-435 (1996).

26. Chun, H. K. et al. Engineering low-aspect ratio carbon nanostructures: nanocups, nanorings, and nanocontainers. ACS Nano 3, 1274-1278 (2009).
27. Jung, H. Y. et al. Transparent, flexible supercapacitors from nano-engineered carbon films. Sci. Rep. 2, 773 (2012).

28. Perdew, J. P. \& Zunger, A. Self-interaction correction to density-functional approximations for many-electron systems. Phys. Rev. B 23, 5048-5079 (1981).

29. Ceperley, D. M. \& Alder, B. J. Ground state of the electron gas by a stochastic method. Phys. Rev. Lett. 45, 566-569 (1980).

30. Soler, J. M. et al. The SIESTA method for $a b$ initio order- $N$ materials simulation. J. Phys.: Condens. Matter 14, 2745-2779 (2002).

31. Troullier, N. \& Martins, J. L. Efficient pseudopotentials for plane-wave calculations. Phys. Rev. B 43, 1993-2006 (1991).

32. Kleinman, L. \& Bylander, D. M. Efficacious Form for Model Pseudopotentials. Phys. Rev. Lett. 48, 1425-1428 (1982)

33. Nosé, S. A molecular dynamics method for simulations in the canonical ensemble. Mol. Phys. 52, 255-268 (1984).

34. Hoover, W. G. Canonical dynamics: Equilibrium phase-space distributions. Phys. Rev. A 31, 1695-1697 (1985).

35. Allen, M. P. \& Tildesley, D. J. Computer Simulation of Liquids. New York: Oxford (1990).

\section{Acknowledgments}

Y.J.J. and H.Y.J. acknowledge the financial support from Fundamental R\&D Program for Core Technology of Materials in the Ministry of Knowledge Economy (MKE), Republic of Korea, and National Science Foundation-CMMI grant (0927088). C.W.A. acknowledges the financial support from Global Frontier grant/MEST (No. 2012054560) and MKE (No.A004600091), Republic of Korea. Y.K.K., S.P. and S.H.K. gratefully acknowledge the financial support from the National Research Foundation of Korea (Grant Nos. 2011-0002456 and 2012-0005146). Some portion of our computational work was done using the resources of the KISTI Supercomputing Center (KSC-2012-C2-19 and KSC-2012-C2-72). P.M.A. acknowledges the financial support from ARL/ARO (No.W911NF). MU was supported by the National Science Foundation DMR CMMT Program (1106214)

\section{Author contributions}

H.Y.J., P.M.A. and Y.J.J. designed experiments. H.C. fabricated the samples and H.C. and C.W.A. performed the TEM experiments. S.P., S.H.K. and Y.K.K. performed the molecular dynamics (MD) simulations and H.Y.J. and M.U. calculated the mean square displacement. H.Y.J. wrote the manuscript, and all authors discussed the results and contributed to revisions.

\section{Additional information}

Supplementary information accompanies this paper at http://www.nature.com/ scientificreports

Competing financial interests: The authors declare no competing financial interests.

How to cite this article: Jung, H.Y. et al. Liquid metal nanodroplet dynamics inside nanocontainers. Sci. Rep. 3, 2588; DOI:10.1038/srep02588 (2013).

(C) (1) This work is licensed under a Creative Commons Attribution-

BY NC SA NonCommercial-ShareAlike 3.0 Unported license. To view a copy of this license, visit http://creativecommons.org/licenses/by-nc-sa/3.0 